\title{
Effect of the nucleon-delta interaction on properties of the three-nucleon ground state
}

\author{
M. T. Peña \\ Centro de Física Nuclear da Universidade de Lisboa, Instituto Nacional de Investigação Científica, \\ 1699 Lisboa Codex, Portugal
}

\author{
H. Henning and P. U. Sauer \\ Theoretical Physics, University Hannover, 3000 Hannover, Germany
}

(Received 30 May 1990)

\begin{abstract}
Wave-function components containing a single $\Delta$ isobar are included in the calculation of the three-nucleon bound state. In extension to previous work, a nucleon- $\Delta$ potential based on meson exchange is incorporated in the interaction models. The interaction models are constructed phase equivalent with the purely nucleonic Paris and Bonn one boson exchange potential in $q$ space (OBEPQ) potentials. The nucleon- $\Delta$ potential yields an additional contribution of the order of $0.1-0.2 \mathrm{MeV}$ to the effective three-nucleon force. Its effect on the electromagnetic properties of the three-nucleon bound state is also considered and found to be small.
\end{abstract}

\section{INTRODUCTION}

The $\Delta$-isobar approach, which Ref. 1 adopts for calculating properties of the three-nucleon ground state, is extended. The approach accounts for $\Delta$-isobar degrees of freedom explicitly. The corresponding components of the nuclear wave function are generated through twobaryon transition potentials which convert one nucleon into a $\Delta$ isobar by internal nucleonic excitation. The force model to be considered is diagrammatically defined in Fig. 1. Process (b) and its Hermitian conjugate yield the $\Delta$-isobar excitation and deexcitation. The nucleon- $\Delta$ interaction is comprised of the direct process (c) and the exchange process (d).

In many-nucleon systems, e.g., in the three-nucleon ground state, the explicit $\Delta$-isobar excitation yields effective energy-dependent two-nucleon and threenucleon forces as shown in Figs. 2 and 3. The consistency between them is the beauty of the $\Delta$-isobar approach. Existing realizations of the $\Delta$-isobar approach are, however, still limited on four counts.

(1) The Hilbert space, adopted for describing nuclear structure and nuclear reactions, allows for single $\Delta$-isobar

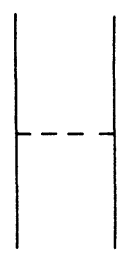

(a)

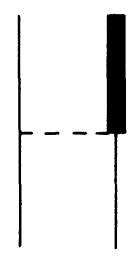

(b)

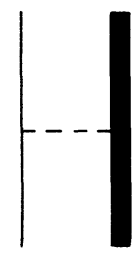

(C)

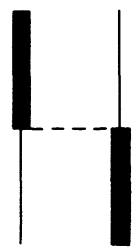

(d)
FIG. 1. Force model with nucleon and $\Delta$-isobar degrees of freedom. A thin vertical line denotes a nucleon, a thick one a $\Delta$ isobar. The Hermitian conjugate process to (b) describing $\Delta$ isobar deexcitation is diagramatically not shown. excitation only. The omission of multiple $\Delta$-isobar excitation can be well motivated ${ }^{2}$ by an analysis of the inelasticity in two-nucleon scattering at intermediate energies up to $500 \mathrm{MeV}$ in the c.m. system. However, that omission is rather imposed than a natural consequence of dynamic structure, and it therefore remains an unaesthetic feature of the force model of Fig. 1. In fact, first attempts $^{3}$ to prove dynamically the smallness of multiple $\Delta$-isobar excitations have not been successful yet.

(2) $\Delta$-isobar excitation and deexcitation is the dominant mechanism for pion production and absorption at intermediate energies. The considered force model, which assumes a stable $\Delta$ isobar with fixed mass in a bound-state calculation, should be derived from its extended versions $\mathrm{s}^{2,4}$ which account for the dynamic structure of the $\Delta$ as pion-nucleon resonance and which are employed when accounting for the two-nucleon system above pion threshold. That relation has only partially been given in Ref. 5 .

(3) The nucleon- $\Delta$ interaction of the processes (c) and (e) in Fig. 3 has been left out in the previous calculation ${ }^{1}$ for the three-nucleon ground state.

(4) $\Delta$-isobar excitation provides only one, though im-

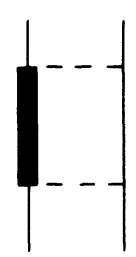

(a)

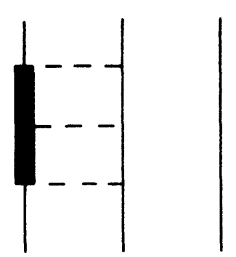

(b)
FIG. 2. Contributions to the effective two-nucleon interaction in the three-nucleon system. Processes up to third order in potentials are shown. Process (b) is due to the nucleon- $\Delta$ interaction and was left out in Ref. 1. 


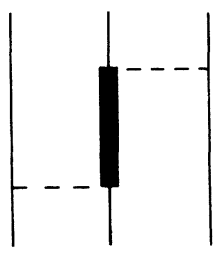

(a)

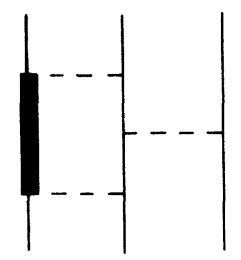

(b)

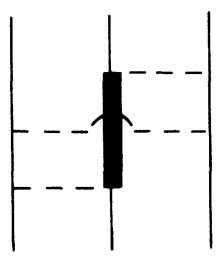

(d)

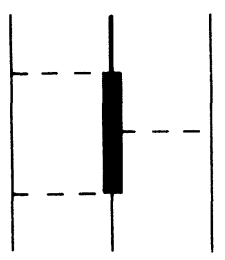

(C)

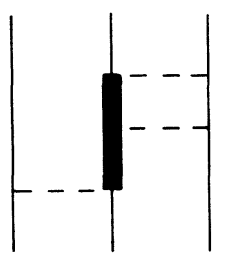

(e)

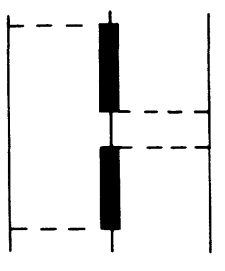

(f)

FIG. 3. Contributions to the effective three-nucleon interaction in the three-nucleon system. Processes up to third order in potentials are shown by the diagrams (a)-(e). Processes (c) and (e) are due to the nucleon- $\Delta$ interaction and were left out in Ref. 1 . Process (d) is an exchange contribution to (b), (e) an exchange contribution to (c). Process ( $f$ ) is of fourth order in the potential. In the calculational step, which sums up potential interactions into a transition matrix, the processes (c) and (f) get lumped together.

portant, mechanism for the three-nucleon force. In contrast, other treatments ${ }^{6,7}$ of the three-nucleon force attempt to accommodate physics in their instantaneous forms, e.g., additional contributions from nonresonant pion-nucleon scattering; the prize to be paid for the richer physics is usually a lack of consistency between twonucleon and three-nucleon forces. The different approaches do not see quantitatively ${ }^{1,8-10}$ the same effect on the three-nucleon binding. It still remains to be studied to what extent the observed discrepancy between results arises from the technically different treatment of the same physics processes and to what extent it arises from the different physics content of the employed threenucleon forces.

The present paper includes the nucleon- $\Delta$ interaction and thereby removes limitation (3) from applications of the force model of Fig. 1. Section II describes the parametrization of the nucleon- $\Delta$ interaction. Section III recalls the technical apparatus adopted for calculating the properties of the three-nucleon ground state. Section IV discusses the results and gives conclusions. Preliminary results were reported in Ref. 11.

\section{PARAMETRIZATION OF THE NUCLEON- $\Delta$ POTENTIAL}

Reference 1, the work that is to be extended in this paper, left out the nucleon- $\Delta$ interaction. However, it did not leave it out on technical grounds. The calculational apparatus developed there was well equipped to include it without complication. It was left out because the nucleon- $\Delta$ potential was hardly known theoretically and the renormalization scheme for preserving the approximate phase equivalence of the extended coupled-channel force model of Fig. 1 with a well-fitted realistic singlechannel nucleon-nucleon potential was poorly tested. But phase equivalence is essential ${ }^{12}$ for any meaningful comparison of results arising from force models with and without $\Delta$ isobar. When the nucleon- $\Delta$ interaction is parametrized, the multichannel potential $v_{a b}$ with $a, b=0,1$ has to be reconsidered as a whole. The notation of Ref. 1 is adopted. The label 1 refers to the twonucleon channel, the label 0 to the one with a $\Delta$ isobar.

The transition potentials $v_{01}$ and $v_{10}$ for $\Delta$-isobar excitation and deexcitation are parametrized as in force model $A 2$ of Ref. 1 through pion $(\pi)$ and rho $(\rho)$ exchange, i.e.,

$$
\begin{aligned}
& v_{01}=\sum_{\alpha=\pi \rho} v_{01}(\alpha), \\
& v_{10}=v_{01}^{+} .
\end{aligned}
$$

In momentum space, the meson contributions take the form

$$
\begin{aligned}
\left\langle\mathbf{p}^{\prime}\left|v_{01}(\pi)\right| \mathbf{p}\right\rangle= & \frac{1}{(2 \pi)^{3}}(-)\left(\frac{g \pi}{2 m_{N}}\right)^{2} \frac{f \pi \Delta N}{f \pi} \frac{\Lambda_{\pi}^{2}-m_{\pi}^{2}}{\Lambda_{\pi}^{2}+\left(\mathbf{p}^{\prime}-\mathbf{p}\right)^{2}}\left[1+P_{12}\right] \frac{\tau(1) \cdot \tau_{\Delta N}(2)}{\left(\mathbf{p}^{\prime}-\mathbf{p}\right)^{2}+m_{\pi}^{2}} \boldsymbol{\sigma}(1) \cdot\left(\mathbf{p}^{\prime}-\mathbf{p}\right) \boldsymbol{\sigma}_{\Delta N}(2) \cdot\left(\mathbf{p}^{\prime}-\mathbf{p}\right), \\
\left\langle\mathbf{p}^{\prime}\left|v_{01}(\rho)\right| \mathbf{p}\right\rangle= & \frac{1}{(2 \pi)^{3}}(-)\left(\frac{g \rho}{2 m_{N}}\right)^{2} \frac{f \pi \Delta N}{f_{\pi}} \frac{\Lambda_{\rho}^{2}-m_{\rho}^{2}}{\Lambda_{\rho}^{2}+\left(\mathbf{p}^{\prime}-\mathbf{p}\right)^{2}}\left[1+P_{12}\right] \frac{\tau(1) \cdot \tau_{\Delta N}(2)}{\left(\mathbf{p}^{\prime}-\mathbf{p}\right)^{2}+m_{\rho}^{2}} \\
& \times\left\{\left(1+\kappa_{\rho}\right)^{2}\left[\boldsymbol{\sigma}(1) \times\left(\mathbf{p}^{\prime}-\mathbf{p}\right)\right] \cdot\left[\sigma_{\Delta N}(2) \times\left(\mathbf{p}^{\prime}-\mathbf{p}\right)\right]+3\left(1+4 / 3 \kappa_{\rho}\right) \sigma_{\Delta N}(2) \cdot i\left(\mathbf{p}^{\prime} \times \mathbf{p}\right)\right\} .
\end{aligned}
$$


The nucleon- $\Delta$ potential $v_{00}$ has a direct and an exchange part according to Figs. 1(c) and 1(d), $v_{00}^{d}$ and $v_{00}^{e}$, respectively. Both pieces are based on meson exchange, i.e.,

$$
\begin{aligned}
& v_{00}=v_{00}^{d}+v_{00}^{e}, \\
& v_{00}^{d}=\sum_{\alpha=\pi \rho \sigma \omega} v_{00}^{d}(\alpha), \\
& v_{00}^{e}=\sum_{\alpha=\pi \rho} v_{00}^{e}(\alpha) .
\end{aligned}
$$

Since the $\Delta$ isobar has isospin $\frac{3}{2}$, only the isovector mesons $\pi$ and $\rho$ contribute to the exchange part, whereas all considered mesons, $\pi, \rho$, sigma $(\sigma)$, and omega $(\omega)$ contribute to the direct part. The different meson contributions are

$$
\begin{aligned}
& \left\langle\mathbf{p}^{\prime}\left|v_{00}^{d}(\pi)\right| \mathbf{p}\right\rangle=\frac{1}{(2 \pi)^{3}}(-)\left(\frac{g \pi}{2 m_{N}}\right)^{2}\left(\frac{1}{25}\right)^{1 / 2} \frac{\Lambda_{\pi}^{2}-m_{\pi}^{2}}{\Lambda_{\pi}^{2}+\left(\mathbf{p}^{\prime}-\mathbf{p}\right)^{2}}\left[1+P_{12}\right] \frac{\tau(1) \cdot \tau_{\Delta \Delta}(2)}{\left(\mathbf{p}^{\prime}-\mathbf{p}\right)^{2}+m_{\pi}^{2}} \boldsymbol{\sigma}(1) \cdot\left(\mathbf{p}^{\prime}-\mathbf{p}\right) \boldsymbol{\sigma}_{\Delta \Delta}(2) \cdot\left(\mathbf{p}^{\prime}-\mathbf{p}\right), \\
& \left\langle\mathbf{p}^{\prime}\left|v_{00}^{e}(\pi)\right| \mathbf{p}\right\rangle=\frac{1}{(2 \pi)^{3}}(-)\left(\frac{g \pi}{2 m N}\right)^{2}\left(\frac{f \pi \Delta N}{f \pi}\right)^{2} \frac{\Lambda_{\pi}^{2}-m_{\pi}^{2}}{\Lambda_{\pi}^{2}+\left(\mathbf{p}^{\prime}-\mathbf{p}\right)^{2}} \\
& \times\left[1+P_{12}\right] \frac{\tau_{\Delta N}(1) \cdot \tau_{\Delta N}^{+}(2)}{\left(\mathbf{p}^{\prime}-\mathbf{p}\right)^{2}+m_{\pi}^{2}} \boldsymbol{\sigma}_{\Delta N}(1) \cdot\left(\mathbf{p}^{\prime}-\mathbf{p}\right) \boldsymbol{\sigma}_{\Delta N}^{+}(2) \cdot\left(\mathbf{p}^{\prime}-\mathbf{p}\right), \\
& \left\langle\mathbf{p}^{\prime}\left|v_{00}^{d}(\sigma)\right| \mathbf{p}\right\rangle=\frac{1}{(2 \pi)^{3}}(-)\left(\frac{g \sigma}{2 m_{N}}\right)^{2} \frac{\Lambda_{\sigma}^{2}-m_{\sigma}^{2}}{\Lambda_{\sigma}^{2}+\left(\mathbf{p}^{\prime}-\mathbf{p}\right)^{2}}\left[1+P_{12}\right] \frac{1}{\left(\mathbf{p}^{\prime}-\mathbf{p}\right)^{2}+m_{\pi}^{2}}\left\{4 m_{N}^{2}-\left(\boldsymbol{\sigma}(1)+\boldsymbol{\sigma}_{\Delta \Delta}(2)\right) \cdot i\left(\mathbf{p}^{\prime} \times \mathbf{p}\right)\right\}, \\
& \left\langle\mathbf{p}^{\prime}\left|v_{00}^{d}(\rho)\right| \mathbf{p}\right\rangle=\frac{1}{(2 \pi)^{3}}\left[\frac{g \rho}{2 m_{N}}\right]^{2} \frac{\Lambda_{\rho}^{2}-m_{\pi}^{2}}{\Lambda_{\rho}^{2}+\left(\mathbf{p}^{\prime}-\mathbf{p}\right)^{2}}\left[1+P_{12}\right] \frac{\tau(1) \cdot \tau_{\Delta \Delta}(2)}{\left(\mathbf{p}^{\prime}-\mathbf{p}\right)^{2}+m_{\rho}^{2}} \\
& \times\left\{4 m_{N}^{2}-\left(\frac{1}{25}\right)^{1 / 2}(1+\kappa \rho)^{2}\left[\boldsymbol{\sigma}(1) \times\left(\mathbf{p}^{\prime}-\mathbf{p}\right)\right] \cdot\left[\sigma_{\Delta \Delta}(2) \times\left(\mathbf{p}^{\prime}-\mathbf{p}\right)\right]\right. \\
& \left.+3(1+4 / 3 \kappa \rho)\left[\sigma(1)+\left(\frac{1}{25}\right)^{1 / 2} \sigma_{\Delta \Delta}(2)\right] \cdot i\left(\mathbf{p}^{\prime} \times \mathbf{p}\right)\right\}, \\
& \left\langle\mathbf{p}^{\prime}\left|v_{00}^{e}(\rho)\right| \mathbf{p}\right\rangle=\frac{1}{(2 \pi)^{3}}(-)\left(\frac{g \rho}{2 m N}\right)^{2}\left(\frac{f \pi \Delta N}{f \pi}\right)^{2} \frac{\Lambda_{\rho}^{2}-m_{\rho}^{2}}{\Lambda_{\rho}^{2}+\left(\mathbf{p}^{\prime}-\mathbf{p}\right)^{2}} \\
& \times\left[1+P_{12}\right] \frac{\tau_{\Delta N}(1) \cdot \tau_{\Delta N}^{+}(2)}{\left(\mathbf{p}^{\prime}-\mathbf{p}\right)^{2}+m_{\rho}^{2}}\left(1+\kappa_{\rho}\right)^{2}\left[\boldsymbol{\sigma}_{\Delta N}(1) \times\left(\mathbf{p}^{\prime}-\mathbf{p}\right)\right] \cdot\left[\boldsymbol{\sigma}_{\Delta N}^{+}(2) \times\left(\mathbf{p}^{\prime}-\mathbf{p}\right)\right], \\
& \left\langle\mathbf{p}^{\prime}\left|v_{00}^{d}(\omega)\right| \mathbf{p}\right\rangle=\frac{1}{(2 \pi)^{3}}\left[\frac{g \omega}{2 m_{N}}\right)^{2} \frac{\Lambda_{\omega}^{2}-m_{\pi}^{2}}{\Lambda_{\omega}^{2}+\left(\mathbf{p}^{\prime}-\mathbf{p}\right)^{2}}\left[1+P_{12}\right] \frac{1}{\left(\mathbf{p}^{\prime}-\mathbf{p}\right)^{2}+m_{\omega}^{2}} \\
& \times\left\{4 m_{N}^{2}-\left[\boldsymbol{\sigma}(1) \times\left(\mathbf{p}^{\prime}-\mathbf{p}\right)\right] \cdot\left[\sigma_{\Delta \Delta}(2) \times\left(\mathbf{p}^{\prime}-\mathbf{p}\right)\right]+3\left[\boldsymbol{\sigma}(1)+\sigma_{\Delta \Delta}(2)\right] \cdot i\left(\mathbf{p}^{\prime} \times \mathbf{p}\right)\right\} .
\end{aligned}
$$

In Eqs. (2.2), (2.4)-(2.7) $\sigma(i)[\tau(i)]$ is the spin [isospin] operator of nucleon $i, \sigma_{\Delta N}(i)\left[\tau_{\Delta N}(i)\right]$ denotes the transition spin [isospin] from nucleon to $\Delta$ isobar, whereas $\sigma_{\Delta \Delta}(i)\left[\tau_{\Delta \Delta}(i)\right]$ refers to the $\Delta$ isobar; their respective reduced matrix elements are $\sqrt{6}, 2$ and $2 \sqrt{15} ; m_{\alpha}$ denotes the meson mass, $m_{N}$ the average nucleonic mass with $m_{N} c^{2}=938.92 \mathrm{MeV}$ and $m_{\Delta}$ the fixed mass of the $\Delta$ isobar with $m_{\Delta} c^{2}=1232 \mathrm{MeV}$. The constant $\Lambda_{\alpha}$ is a regularizing cut-off mass. The operator $P_{12}$ denotes the interchange of the two baryons. All contributions to the nucleon- $\Delta$ potential are local. It can therefore be cast into the following configuration-space form:

$$
\begin{aligned}
& \left\langle\mathbf{r}^{\prime}\left|v_{00}^{d}+v_{00}^{e}\right| \mathbf{r}\right\rangle=\delta\left(\mathbf{r}^{\prime}-\mathbf{r}\right)\left[v_{00}^{d}(\mathbf{r})+v_{00}^{e}(\mathbf{r})\right], \\
& v_{00}^{d}(\mathbf{r})=\left[1+P_{12}\right] \tau(1) \cdot \tau_{\Delta \Delta}(2)\left\{v_{C}^{d}(r)+v_{\sigma}^{d}(r) \boldsymbol{\sigma}(1) \cdot \sigma_{\Delta \Delta}(2)+v_{T}^{d}(r) S_{N \Delta}^{N \Delta}(1,2)\right. \\
& \left.+v_{L S}^{d}(r) \frac{1}{2}\left[\boldsymbol{\sigma}(1)+\boldsymbol{\sigma}_{\Delta \Delta}(2)\right] \cdot \mathbf{L}+v_{L S a}^{d}(r) \frac{1}{2}\left[\boldsymbol{\sigma}(1)-\boldsymbol{\sigma}_{\Delta \Delta}(2)\right] \cdot \mathbf{L}\right\}, \\
& v_{00}^{e}(\mathbf{r})=\left[1+P_{12}\right] \tau_{\Delta N}(1) \cdot \tau_{\Delta N}^{+}(2)\left[v_{\sigma}^{e}(r) \sigma_{\Delta N}(1) \cdot \sigma_{\Delta N}^{+}(2)+v_{T}^{e}(r) S_{N \Delta}^{\Delta N}(1,2)\right],
\end{aligned}
$$


where the tensor operators are defined as

$$
S_{N \Delta}^{N \Delta}(1,2)=3 \boldsymbol{\sigma}(1) \cdot \hat{\mathbf{r}} \boldsymbol{\sigma}_{\Delta \Delta}(2) \cdot \hat{\mathbf{r}}-\boldsymbol{\sigma}(1) \cdot \sigma_{\Delta \Delta}(2)
$$

and

$$
S_{N \Delta}^{\Delta N}(1,2)=3 \sigma_{\Delta N}(1) \cdot \hat{\mathbf{r}} \sigma_{\Delta N}^{+} \cdot \hat{\mathbf{r}}-\sigma_{\Delta N}(1) \cdot \boldsymbol{\sigma}_{\Delta N}^{+}(2)
$$

and $\mathbf{L}$ denotes the orbital angular momentum. The spinorbit part has two contributions, i.e., $v_{L S}^{d}(r)$ and $v_{L S a}^{d}(r)$. The usual one, $v_{L S}^{d}(r)$, depends on the total spin $\frac{1}{2}\left[\boldsymbol{\sigma}(1)+\sigma_{\Delta \Delta}(2)\right]$, whereas $v_{L S a}^{d}(r)$ goes with the spin difference.

The meson-exchange contributions $(2.2),(2.4)-(2.7)$ to the transition potentials and to the nucleon- $\Delta$ interaction are not derived from field-theoretic Lagrangians describing the fundamental coupling of mesons to the $\Delta$ isobar. Instead, the $\Delta$ isobar is assumed to behave qualtitatively like a nucleon, and known two-nucleon meson-exchange forms are simply transcribed to the $\Delta$ isobar, taking care of the difference in spin, isospin, and coupling strength. This transcription is admitedly a poor approach and particularly shakey for the $\sigma$ meson which does not have a convincing theoretical basis. The employed meson masses ana the meson couplings to the nucleon are listed in Table I. Those parameters, connected with $\pi$ and $\rho$ exchange and also arising in the transition potential $v_{01}$, have already been used in Ref 1 , the others are a mixed bag taken from various sources. ${ }^{13,14}$ The rescaling of the transition potential and of the nucleon- $\Delta$ exchange potential is governed by the ratio of the pion pseudovector coupling strengths. The ratio arises in the form $f_{\pi \Delta N} / f_{\pi}$, where $f_{\pi}$ is the pion coupling strength to the nucleon and $f_{\pi \Delta N}$ is the coupling strength for the transition from nucleon to $\Delta$ isobar. The value $\left(f_{\pi \Delta N} / f_{\pi}\right)^{2}=0.35 / 0.08$ is chosen as in Ref. 1 . The couplings, newly required for the direct nucleon- $\Delta$ potential, are scaled according to quark counting rules as in Ref. 15 . The rescaling factors are incorporated in the potential forms $(2.4)-(2.7)$. The

TABLE I. Meson parameters employed in the construction of the potentials $v_{01}$ and $v_{00}$. All given coupling constants refer to the coupling with nucleons. The parameters related to the $\pi$ and $\rho$ mesons are those of Ref. 1 and motivated there. The parameters related to the $\sigma$ and $\omega$ mesons are taken from Table IV of Ref. 13. $\kappa_{\alpha}$ is the ratio of the tensor to vector coupling for the vector mesons $\rho$ and $\omega$. The pion pseudovector coupling constant $f_{\pi}$ with $f_{\pi}^{2} / 4 \pi=0.08$ is interchangeably used instead of the pseudoscalar coupling constant $g_{\pi}$, since $f_{\pi}=\left(m_{\pi} /\right.$ $\left.2 m_{N}\right) g_{\pi}$. The change in coupling constants from the purely nucleonic one-boson exchange potential to the transition potential $v_{01}$ and to the nucleon- $\Delta$ potential $v_{00}$ is given explicitly in the listed potential forms of Eqs. (2.2), (2.4) - (2.7).

\begin{tabular}{lclcc}
\hline$\alpha$ & $g_{\alpha}^{2} / 4 \pi$ & $\kappa_{\alpha}$ & $m_{\alpha}(\mathrm{MeV})$ & $\Lambda_{\alpha}(\mathrm{GeV})$ \\
\hline$\pi$ & 14.4 & & 138 & 1.2 \\
$\sigma$ & 5.7 & & 500 & 1.2 \\
$\rho$ & 0.55 & 6.6 & 760 & 1.2 \\
$\omega$ & 14.0 & 0 & 780 & 1.2 \\
\hline \hline
\end{tabular}

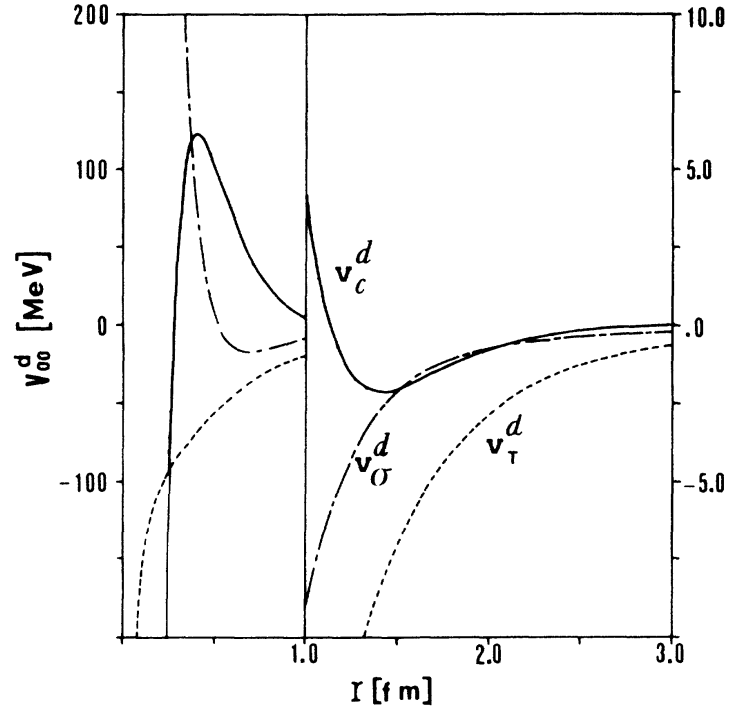

FIG. 4. Direct nucleon- $\Delta$ potential $v_{00}^{d}(\mathbf{r})$ in configuration space as function of the distance $r$ in $\mathrm{fm}$ between the two baryons. It is split up into contributions of different spin structure according to Eq. (2.8a). Note the difference in scales for the potential below and above $1 \mathrm{fm}$.

factor is $\left(\frac{1}{25}\right)^{1 / 2}$ for contributions depending on spin and isospin, the factor is one for all others. The resulting contributions to the local nucleon- $\Delta$ potential are displayed in Figs. 4 and 5. The transcription of the twonucleon meson-exchange potential to the transition po-

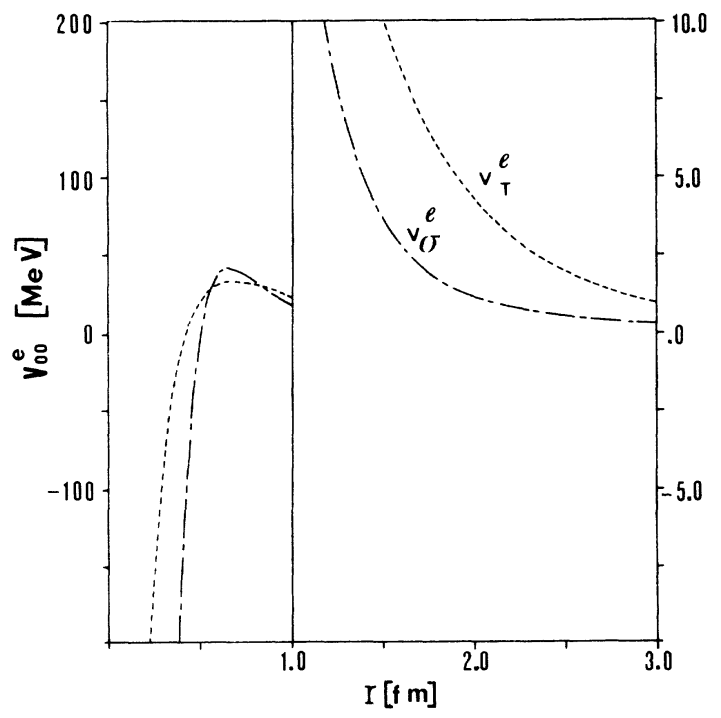

FIG. 5. Exchange nucleon- $\Delta$ potential $v_{00}^{e}(\mathbf{r})$ in configuration space as function of the distance $r$ in $\mathrm{fm}$ between the two baryons. It is split up into contributions of different spin structure according to Eq. (2.8b). Note the difference in scales for the potential below and above $1 \mathrm{fm}$. 
tential $v_{01}$ and to the nucleon- $\Delta$ potential $v_{00}$ yields the traditional ranges for all meson exchanges. In this way, $v_{00}$ is used. In contrast, the force model $A 2$ of Ref. 1 attempts to account for the fact that the transition to a nucleon- $\Delta$ state also changes the two-baryon rest mass by employing different ranges $^{16}$ for the two structurely different time orders of meson exchange. The transition potential $v_{01}$ is used in this paper as in the force model $A$ 2, a fact, not made explicit in Eq. (2.2). Furthermore, the spin-orbit part of Eq. (2.2b) was left out in Ref. 1. This is the reason why the spin-orbit contributions to the transition potential $v_{01}$ and to the direct nucleon- $\Delta$ potential $v_{00}$ are also left in the calculations of this paper. The meson parameters of the nucleon- $\Delta$ interaction are not fitted to any experimental data and are therefore unknown as for Ref. 1. However, the nucleon- $\Delta$ interaction has a quantitative effect on pion-deuteron elastic scattering in particular ${ }^{17}$ and on the two-nucleon system above pion threshold in general. ${ }^{18}$ Thus, in future those data could calibrate the nucleon- $\Delta$ interaction.

The nucleonic part $v_{11}$ of the force model has to be chosen with a view on the transition to and on the interaction in the nucleon- $\Delta$ channel, i.e., on the potentials $v_{01}$ and $v_{00}$. It is to be parametrized such that the complete force model accounts for the experimental data in isospin-triplet partial waves, i.e., for $T=1$, as well as realistic purely nucleonic potentials $v_{p}$ do. It is therefore chosen as

$$
v_{11}=v_{p}-v_{10} \frac{1}{0-k_{0}-v_{00}} v_{01} .
$$

In isospin singlet partial waves, which lack nucleon- $\Delta$ channels, i.e. for $T=0, v_{11}$ is identified with $v_{p}$. In Eq. (2.9) $k_{a}$ is the kinetic-energy operator of relative motion with the inclusion of the rest-mass differences $\left(m_{\Delta}-m_{N}\right) c^{2}$ in the channel 0 with a $\Delta$ isobar. Thus, $k_{a}$ is different in purely nucleonic channels 1 and in channels 0 with a $\Delta$ isobar. It defines the free resolvent by $g_{0 a}(\omega)=\left(\omega-k_{a}\right)^{-1}$. As a result, the two-nucleon transition matrix $t_{11}(\omega)$ satisfies a Lippmann-Schwinger equation of the form

$$
\begin{aligned}
t_{11}(\omega)= & {\left[v_{11}+v_{10} \frac{1}{\omega-k_{0}-v_{00}} v_{01}\right] } \\
& \times\left[1+\frac{1}{\omega-k_{1}} t_{11}(\omega)\right], \\
t_{11}(\omega)= & {\left[v_{p}+v_{10}\left(\frac{1}{\omega-k_{0}-v_{00}}-\frac{1}{0-k_{0}-v_{00}}\right] v_{01}\right] } \\
& \times\left[1+\frac{1}{\omega-k_{1}} t_{11}(\omega)\right]
\end{aligned}
$$

Equation (2.10) is just an artful replacement of the traditional multichannel Lippmann-Schwinger equation

$$
t_{a b}(\omega)=v_{a b}+\sum_{c} v_{a c} g_{0 c}(\omega) t_{c b}(\omega)
$$

which is used for all practical calculations of $t(\omega)$. By construction, $t_{11}(\omega)$ is identical to the two-nucleon transition matrix of the purely nucleonic reference potential $v_{p}$ at energy $\omega=0$. However, approximate phase equivalence is even ensured at all energies up to $165 \mathrm{MeV}$ in the c.m. system, at which $v_{p}$ is fitted. In fact, two force models will be employed in this paper. They are based on the Paris potential ${ }^{19}$ and on the Bonn potential one boson exchange potential in $Q$ space (OBEPQ) (Ref. 14) as nucleonic reference potentials $v_{p}$. For example, in case of the Paris potential, the phase shifts of the considered force model in ${ }^{1} S_{0}$, the isospin-triplet partial wave most important for nuclear binding, differ from those of the Paris potential by $0.5^{\circ}$ at most. The corresponding effective range parameters, i.e., $a=-17.6 \mathrm{fm}$ and $r_{0}=2.81 \mathrm{fm}$, show a similar agreement with those of the Paris potential, $a=-17.61 \mathrm{fm}$ and $r_{0}=2.88 \mathrm{fm}^{19}$. Our numerical determination of the effective parameters is not really perfect, though the observed slight deviation in the range parameter $r_{0}$ appears to be beyond doubt. Nevertheless, the required phase equivalence of the considered force models with their purely nucleonic reference potentials $v_{p}$ is satisfactorily achieved by the choice (2.9).

\section{CALCULATION SCHEME}

The three-nucleon ground state is described by the Hamiltonian

$$
H=K+\sum_{\alpha} v(\alpha),
$$

where $K$ is the three-baryon kinetic-energy operator and $v(\alpha)$ the multichannel potential between the baryon pair $(\beta \gamma)$ defined in Sec. II for the pair $3=(12)$. The free resolvent is $G_{0}(\Omega)=(\Omega-K)^{-1}$. It is channel-dependent, though channel-diagonal. The technical apparatus for solving the Faddeev equations

$$
\begin{aligned}
& |\psi\rangle=G_{0}\left(E_{B}\right) T\left(E_{B}\right)\left[P_{123}+P_{321}\right]|\psi\rangle, \\
& \left|\psi_{B}\right\rangle=\left[1+P_{123}+P_{321}\right]|\Psi\rangle,
\end{aligned}
$$

is taken over unchanged from Ref. 1. The Faddeev amplitudes $|\psi(\alpha)\rangle=G_{0}\left(E_{B}\right) v(\alpha)\left|\psi_{B}\right\rangle$ yield the bound-state wave function $\left|\psi_{B}\right\rangle$ according to Eq. (3.2b) with the three-nucleon binding energy $E_{B}$. Their label $\alpha$ is left out in Eq. (3.2a), since due to the identity of particles all Faddeev amplitudes can be obtained from a single representative one by permutations, $P_{123}$ and $P_{321}$ being the cyclic and anticyclic permutation operators of three particles, respectively. All nucleonic components in the boundstate wave function are properly antisymmetrized. Since the transition potential to single $\Delta$-isobar configurations is symmetric in all particles, only full antisymmetrized configurations couple. Thus, the baryons in all channels can be treated as identical, though the $\Delta$ isobar is distinct from the nucleon.

The operator $T(\Omega)$ is the two-baryon transition matrix $t(\omega)$ of Sec. II, embedded in three-baryon Hilbert space, the available energies $\Omega$ and $\omega$ being related by the fact that $T(\Omega)$ propagates a spectator baryon besides the two interacting ones. The transition matrix $T(\Omega)$ or $t(\omega)$ is 
TABLE II. Partial waves up to total angular momentum $I=2$. The partial waves in which the two-baryon potential acts are split according to isospin $T$. Those nucleon- $\Delta$ partial waves which do not couple to any nucleonic ones are listed in parentheses; there are some with isospin $T=2$, e.g., ${ }^{3} S_{1}$, which are not given in the table.

\begin{tabular}{llll}
\hline \hline & $I$ & $N N$ & \multicolumn{1}{c}{$N \Delta$} \\
\hline$T=0$ & 1 & ${ }^{3} S_{1}{ }^{3} D_{1}$ & \\
& & ${ }^{1} P_{1}$ & \\
& 2 & ${ }^{3} D_{2}$ & \\
& 0 & ${ }^{1} S_{0}$ & ${ }^{5} D_{0}$ \\
& 1 & ${ }^{3} P_{0}$ & ${ }^{3} P_{0}$ \\
& & & $\left({ }^{3} S_{1}{ }^{3} D_{1}{ }^{5} D_{1}\right)$ \\
& & ${ }^{3} P_{1}$ & ${ }^{3} P_{1}{ }^{5} P_{1}{ }^{5} F_{1}$ \\
& 2 & ${ }^{1} D_{2}$ & ${ }^{5} S_{2}{ }^{3} D_{2}{ }^{5} D_{2}{ }^{5} G_{2}$ \\
& & ${ }^{3} P_{2}{ }^{3} F_{2}$ & ${ }^{3} P_{2}{ }^{5} P_{2}{ }^{3} F_{2}{ }^{5} F_{2}$ \\
\hline \hline
\end{tabular}

needed in all its channels, i.e., $T_{11}(\Omega), T_{10}(\Omega), T_{01}(\Omega)$, and $T_{00}(\Omega)$ arise in Eq. (3.2a).

The same basis states with the same truncation on the partial waves of the two-baryon potential and on the considered Faddeev amplitudes are adopted as in Ref. 1. Up to total two-baryon angular momentum $I=2$ the partial waves of the considered force model are listed in Table II. There are nucleon- $\Delta$ partial waves which do not couple to two-nucleon states, but through which the force model contributes to binding in the three-nucleon ground state. Those partial waves are left out in the calculations of this paper. Only the effect of the ${ }^{3} S_{1}$ nucleon- $\Delta$ partial wave with isospin $T=1$, which appears most important since the corresponding nucleonic one is of overwhelming importance, will be studied in one additional calculation.

From the resulting bound-state wave function $\left|\psi_{B}\right\rangle$ the electromagnetic (e.m.) properties of the three-nucleon ground state are calculated. The charge and current operators used are those of Ref. 20.

\section{RESULTS AND CONCLUSIONS}

The properties of the three-nucleon ground state predicted by the force model of Fig. 1 are listed in Table III. The new results of this paper, based on the Paris potential as nucleonic reference potential, are labeled as $A 3$, those based on the Bonn potential OBEPQ as $C 3$. They are compared with results obtained from the nucleonic reference potentials and from their extensions ${ }^{1,22} A 2$ and $C 2$ with $\Delta$ excitation, though without nucleon- $\Delta$ interaction.

The added nucleon- $\Delta$ interaction contributes additional binding, but the resulting energy increase is rather small, about $0.1 \mathrm{MeV}$ for the force model $A 3$. That energy increase is analyzed in detail for $A 3$. Besides a slight redistribution between the dominating processes of twonucleon dispersion $\Delta E_{2}$ of Fig. 2 and of the most impor-

TABLE III. Three-nucleon ground-state properties. The new results of this paper for the force model of Fig. 1 are listed in the columns $A 3$ and $C 3$. They correspond to the Paris potential (Ref. 19) and the Bonn potential OBEPQ (Ref. 14) as purely nucleonic reference potentials, respectively. The results for the Paris potential and its extended version $A 2$ and $\Delta$-isobar excitation though without nucleon- $\Delta$ interaction is taken over from Ref. 1, the corresponding results for the Bonn potential OBEPQ and its extension $C 2$ from Ref. 22. The row labeled $E_{B}$ lists the full triton binding energy, its experimental value being $-8.48 \mathrm{MeV}$, the one labeled $\Delta E_{2}$ the two-nucleon dispersive effect of Fig. 2 on binding, and the one labeled $\Delta E_{3}$ the complete effect of the effective three-nucleon force according to Fig. 3 . In case of the force models $A 2$ and $A 3$ the latter result is split up according to the individual processes of Fig. 3 in the following rows. The contribution from higher-order processes as Fig. 3(f) is also given. Because of the omission of the nucleon- $\Delta$ interaction, the force models $A 2$ and $C 2$ do not receive any contribution from the process of Fig. 3(c). The probabilities $P(\mathcal{L})$ for the nucleonic wave-function components, of different orbital angular momentum $\mathcal{L}$ and symmetry specification $S, S^{\prime}, P$, and $D$ and for the $\Delta$ excitation, i.e., $P(\Delta)$, are also listed. The probabilities are computed from the Faddeev amplitudes. Those resulting from the truncated partial wave expansion of the wave function employed when computing e.m. properties are slightly different. The differences are of the same order of magnitude as in Table 4 of Ref. 1.

\begin{tabular}{crrrrrr}
\hline \hline & Paris & \multicolumn{1}{c}{$A 2$} & \multicolumn{1}{c}{$A 3$} & OBEPQ & \multicolumn{1}{c}{$C 2$} & \multicolumn{1}{c}{$C 3$} \\
\hline$E_{B}(\mathrm{MeV})$ & -7.38 & -7.72 & -7.85 & -8.29 & -8.39 & -8.61 \\
$\Delta E_{2}(\mathrm{MeV})$ & & 0.58 & 0.46 & & 0.69 & 0.52 \\
$\Delta E_{3}(\mathrm{MeV})$ & & -0.92 & -0.93 & & -0.79 & -0.84 \\
Fig. 3(a) & & -0.83 & -0.75 & & & \\
Fig. 3(b) & & -0.02 & -0.02 & & & \\
Fig. 3(c) & & & -0.09 & & & \\
Fig. 3(f) & & -0.07 & -0.07 & & & \\
$P(S)$ & 90.12 & 87.65 & 88.18 & 92.55 & 89.61 & 1.29 \\
$P\left(S^{\prime}\right)$ & 1.40 & 1.27 & 1.24 & 1.24 & 0.06 & 0.05 \\
$P(P)$ & 0.06 & 0.09 & 0.08 & 0.04 & 0.06 & 6.43 \\
$P(D)$ & 8.42 & 8.66 & 8.70 & 6.17 & 6.40 & 2.07 \\
$P(\Delta)$ & & 2.33 & 1.80 & & 2.68 & \\
\hline \hline
\end{tabular}


tant part (a) in the three-nucleon force of Fig. 3, the additional binding basically arises from the process (c) of Fig. 3 , which did not contribute in Refs. 1 and 20. The split of the complete effect $\Delta E_{3}$ of the three-nucleon force into individual contributions is done as in Ref. 1 by additional calculations with truncated sets of Faddeev amplitudes and interaction channels. Unfortunately, the split between the effect from the nucleon- $\Delta$ potential $v_{00}$ according to Fig. 3(c), i.e., $-0.09 \mathrm{MeV}$ in Table III for $A 3$, and the effect from higher-order contributions to the nucleon- $\Delta$ transition matrix $t_{00}(\omega)$ from the transition potential $v_{01}$ according to Fig. 3 (f), i.e., $-0.07 \mathrm{MeV}$ in Table III for $A 3$, cannot be made precise. It is done approximately by assuming that the effect of higher-order contributions is the same for the force models $A 2$ and $A 3$. We consider the given analysis of the small total energy increase for $A 3$ as compared to force model $A 2$ trustworthy. Admittedly, such a small change could also be caused by a lack of exact phase equivalence and, in fact, phase equivalence has not been achieved in a perfect manner as discussed in Sec. III. Phase equivalence shows up strongest in the ${ }^{1} S_{0}$ effective range $r_{0}$, i.e., $r_{0}=2.79$ fm for $A 2$ and $r_{0}=2.81 \mathrm{fm}$ for $A 3$. However, according to Ref. 21 , such an on-shell deviation would yield by itself a binding-energy shift of opposite sign and of smaller size than the one observed.

The found results slightly depend on the meson parameters chosen. In fact, a calculation with the $\sigma$ and $\omega$ parameters of Table IV in Ref. 14 instead, i.e., $g_{\sigma}^{2} / 4 \pi=5.7$, $m_{\sigma} c^{2}=550 \mathrm{MeV}, g_{\omega}^{2} / \pi=20.0, m_{\omega} c^{2}=783 \mathrm{MeV}$, yields a decreased total tritium binding of $-7.80 \mathrm{MeV}$ for force model $A 3$. However, it was checked that a nucleon- $\Delta$ interaction in the isospin-triplet ${ }^{3} S_{1}$ partial wave, which does not couple to purely nucleonic channels, yields only minute additional binding, not changing the results of Table III in the quoted figures. On the other hand, the increase in binding due to the nucleon- $\Delta$ interaction is rather similar for other nucleonic reference potentials as borne out for the Bonn potential OBEPQ by the column C3.

Though the nucleon- $\Delta$ interaction can be strong as Figs. 4 and 5 demonstrate for its configuration-space form, the weight of $\Delta$-isobar configurations in the threenucleon ground state is with approximately $2 \%$ rather modest. Thus, the smallness of its effect on binding is plausible. The same small effect is observed for the e.m. properties. This fact is expected already from Table III, since the additional nucleon- $\Delta$ interaction hardly changes the wave function probabilities $P(\mathcal{L})$. Figure 6 shows results of a calculation for the ${ }^{3} \mathrm{He}$ charge monopole form factor $F_{c}\left(Q^{2}\right)$ and for the ${ }^{3} \mathrm{He}$ magnetic dipole form factor $F_{M}\left(Q^{2}\right)$ based on the force models $A 2$ and $A 3$. The calculation is based on the nonrelativistic operators of Ref. 20. Thus, one-baryon charge operators are employed, whereas the spatial current gets the standard $\pi$ and $\rho$-exchange contributions. In contrast to the presentation of Ref. 20 its successful spatial current operators can be given an entirely nonrelativistic derivation accord-
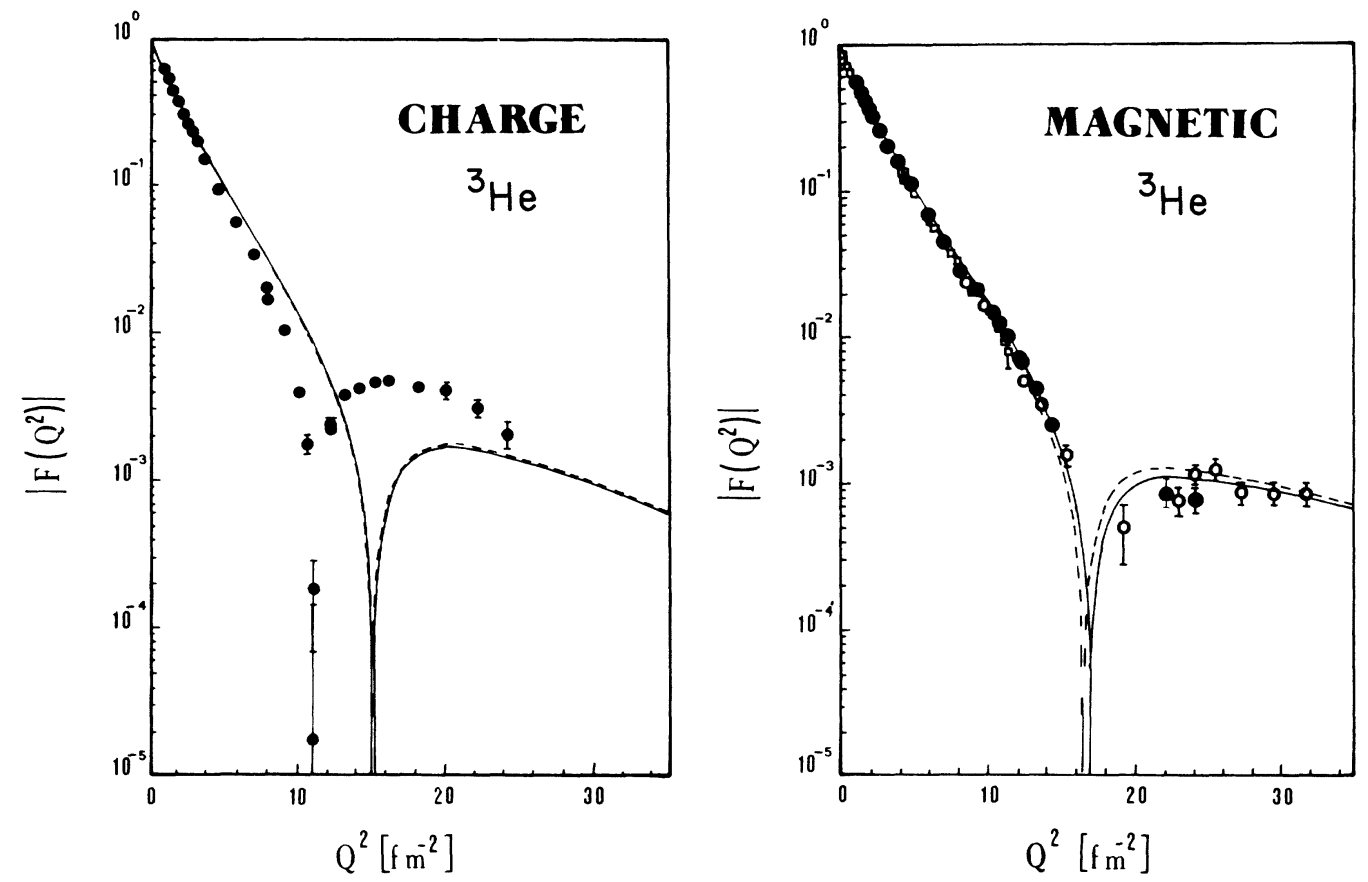

FIG. 6. ${ }^{3} \mathrm{He}$ charge monopole (left) and ${ }^{3} \mathrm{He}$ magnetic dipole form factors as function of momentum transfer $Q^{2}$. The solid line based on force model $A 3$ refers to the calculation of this paper with the inclusion of the nucleon- $\Delta$ interaction, the dashed line based on force model $A 2$ to the result of Ref. 20. The experimental data are taken from Ref. 23, which presents new measurements (solid circle) and uses in addition a special selection of previous data [open circles (Ref. 24), open squares (Ref. 25)] of the magnetic form factor in a new analysis (Ref. 23). Those data points of Ref. 23 at which the magnetic form factor contributes less than 10\% to the cross section are not included in Fig. 6(b). 
ing to Ref. 22. One notes that even the improved description of the three-nucleon force is unable to polarize the nucleonic distribution substantially and to describe the charge form factor without additional twobaryon charge operators of relativistic order any better. In fact, due to the slight increase of binding the position of the diffraction minimum even gets worsened.

Compared with predictions from two-nucleon potentials, since $\Delta$-isobar excitation yields rather little additional binding. This result of Ref. 1, confirmed by this paper, came as a surprise, since $\Delta$-isobar excitation generates a substantial part of the three-nucleon force with pion range and the corresponding instantaneous parametrizations usually provide ample additional binding. ${ }^{8-10}$ It was suspected that the omission of a nucleon- $\Delta$ interaction may be partly held responsible for this surprising fact. In contrast, the present calculation makes the interpretation reached originally in Ref. 1 even firmer: Single $\Delta$-isobar excitation yields a sizable contribution to binding in form of a three-nucleon force, but binding is lost simultaneously by two-nucleon dispersion. The threenucleon force effect is itself reduced by the energy dependence of the $\Delta$-isobar propagation compared to instantaneous approximations and, furthermore, its $\pi$-exchange contribution is screened ${ }^{3}$ by the included $\rho$ exchange.

Though the effect of the nucleon- $\Delta$ interaction on properties of the three-nucleon ground state appears small, it would nevertheless be more satisfying to derive the force model of this paper from its extended versions, ${ }^{2,4}$ those versions which describe the full wealth of the two-nucleon system above pion threshold with its explicit coupling to inelastic channels, and to test the nucleon- $\Delta$ interaction there before using it in the description of the three-nucleon ground state.

\section{ACKNOWLEDGMENTS}

Useful discussions with A. Bulla and H. Garcilazo are gratefully acknowledged. The calculations were done at Regionales Rechenzentrum für Niedersachsen (RRZN) in Hannover and at Instituto Nacional de Investigação Científica (INIC), Lisbon. M.T.P. received financial support from Deutscher Akademischer Austauschdienst (DAAD) which made a stay in Hannover possible during which part of the work was done. This work was funded by the Deutsche Forchungsgemeinschaft (DFG) under Contract Nos. Sa 247/7-1 and Sa 247/9-3.
${ }^{1}$ Ch. Hajduk, P. U. Sauer, and W. Strueve, Nucl. Phys. A405, 581 (1983).

${ }^{2}$ H. Pöpping, P. U. Sauer, and Zhang Xi-Zhen, Nucl. Phys. A474, 557 (1987).

${ }^{3}$ Ch. Hajduk, P. U. Sauer, and Shin Nan Yang, Nucl. Phys. A405, 605 (1983).

${ }^{4}$ T.-S. H. Lee, Phys. Rev. C 29, 195 (1984).

${ }^{5}$ K. Dreissigacker, S. Furui, P. U. Sauer, and R. Machleidt, Nucl. Phys. A375, 334 (1982); H. Pöpping, P. U. Sauer, and Zhang Xi-Zhen, Few Body Problems in Physics, Few Body X Contributed Papers, edited by B. Zeitnitz (North-Holland, Amsterdam, 1984), Vol. II, p. 145.

${ }^{6}$ S. A. Coon, M. D. Scadron, P. C. McNamee, B. R. Barret, D. W. E. Blatt, and B. H. J. McKellar, Nucl. Phys. A317, 242 (1979).

${ }^{7}$ H. T. Coelho, T. K. Das, and M. R. Robilotta, Phys. Rev. C 28, 1812 (1983).

${ }^{8}$ R. B. Wiringa, J. L. Friar, B. F. Gibson, G. L. Payne, and C. R. Chen, Phys. Lett. 143B, 273 (1984); C. R. Chen, G. L. Payne, J. L. Friar, and B. F. Gibson, Phys. Rev. C 33, 1740 (1986).

${ }^{9}$ A. Bömelburg and W. Glöckle, Phys. Rev. C 28, 1812 (1983); A. Bömelburg, ibid. 34, 14 (1986).

${ }^{10} \mathrm{~S}$. Ishikawa and T. Sasakawa, Few-Body Systems 1, 143 (1986).

${ }^{11}$ M. T. Peña, H. Henning, and P. U. Sauer, Contribution to the Few-Body XII Conference, edited by B. K. Jennings (TRIUMF, Vancouver, 1989), p. G50.

${ }^{12}$ Ch. Hajduk and P. U. Sauer, Nucl. Phys. A322, 329 (1979).
${ }^{13}$ K. Erkelenz, Phys. Rev. C 13, 5 (1974).

${ }^{14}$ R. Machleidt, K. Holinde, and Ch. Elster, Phys. Rep. 149, 1 (1987).

${ }^{15}$ G. E. Brown and W. Weise, Phys. Rev. C 22, 281 (1975).

${ }^{16}$ J. W. Durso, M. Saarela, G. E. Brown, and A. D. Jackson, Nucl. Phys. A278, 445 (1977).

${ }^{17}$ E. Ferreira, S. C. Andrade, and H. G. Dosch, Phys. Rev. C 36, 1916 (1987).

${ }^{18} \mathrm{C}$. Alexandrou and B. Blankleider, Paul Scherrer Institute Report PR-89-34, 1990.

${ }^{19}$ M. Lacombe, B. Loiseau, J. M. Richard, R. Vinh Mau, J. Côté, P. Pirès, and R. de Tourreil, Phys. Rev. C 21, 861 (1980).

${ }^{20}$ W. Strueve, Ch. Hajduk, P. U. Sauer, and W. Theis, Nucl. Phys. A465, 651 (1987).

${ }^{21}$ B. F. Gibson and G. J. Stephensen, Jr., Phys. Rev. C 8, 1222 (1973).

${ }^{22}$ F. Gross, H. Henning, P. U. Sauer, and W. Theis, Hannover report, 1990.

${ }^{23}$ Akila Amroun, Ph.D. thesis, Saclay, 1989 (unpublished); S. K. Platchkov, private communcation.

${ }^{24}$ J. M. Cavedon, B. Frois, D. Goutte, M. Huet, Ph. Leconte, J. Martino, X. H. Phan, S. K. Platchkov, S. E. Williamson, W. Boeglin, I. Sick, P. de Witt-Huberts, L. S. Cardman, and C. N. Papanicolas, Phys. Rev. Lett. 49, 986 (1982).

${ }^{25}$ P. C. Dunn, S. B. Kowalski, F. N. Rad, C. P. Sargent, and W. E. Turchinetz, Phys. Rev. C 27, 71 (1983). 